\title{
Estudo numérico da influência de condições de contorno na modelagem computacional de trocador de calor solo-ar
}

Numerical study of the influence of boundary conditions on the computational modeling of earth-air heat exchanger

\author{
M. K. Rodrigues ${ }^{1}$; J. A. Souza ${ }^{1,2}$; J. Vaz ${ }^{2}$; I. C. A. Junior ${ }^{3}$; L. A. O. Rocha ${ }^{1,4}$; \\ R. S. Brum ${ }^{5}$ E. D. dos Santos ${ }^{1,2}$; L. A. Isoldi ${ }^{1,2}$ \\ ${ }^{I}$ Programa de Pós-Graduação em Modelagem Computacional (PPGMC), Universidade Federal do Rio Grande \\ (FURG) , 96.203-900, Rio Grande-RS, Brasil \\ ${ }^{2}$ Escola de Engenharia (EE), Universidade Federal do Rio Grande (FURG), 96.203-900, Rio Grande-RS, Brasil \\ ${ }^{3}$ Instituto Federal de Educação, Ciência e Tecnologia do Rio Grande do Sul (IFRS), 96.201-460, \\ Rio Grande-RS, Brasil \\ ${ }^{4}$ Departamento de Engenharia Mecânica (DEMEC), Universidade Federal do Rio Grande do Sul (UFRGS), \\ 90.050-170, Porto Alegre-RS, Brasil \\ ${ }^{5}$ Departamento de Matemática e Estatística (DME), Universidade Federal de Pelotas (UFPel), 96.010-900, \\ Capão do Leão-RS, Brasil
}

*michel.professor@yahoo.com.br

(Recebido em 22 de outubro de 2014; aceito em 07 de março de 2015)

O trocador de calor solo-ar (TCSA) é um dispositivo utilizado para a melhoria da condição térmica de ambientes construídos, contribuindo na diminuição no consumo de energia elétrica dos equipamentos tradicionais de condicionamento de ar. Assim, os objetivos deste trabalho são de avaliar a influência da condição de contorno térmica de fluxo prescrito nulo no potencial térmico (PT) do TCSA, que é imposta nas superfícies laterais do domínio computacional, e de estudar a efeito do tipo de condição de contorno térmica imposta na superfície inferior do domínio computacional no tempo de processamento da simulação numérica de um TCSA. Para isso, um modelo computacional, validado e verificado, desenvolvido no software FLUENT, que é baseado no Método dos Volumes Finitos (MVF), foi empregado. Os resultados mostraram que o PT das instalações de TCSA analisadas é afetado para um espaçamento horizontal, entre o duto e a parede do domínio computacional, inferior a 2,00 m. Além disso, foi observado que a utilização da condição de contorno de temperatura prescrita imposta na superfície inferior do domínio computacional contribuiu para a redução do tempo de processamento da simulação numérica em $18 \%$, quando comparada com a utilização da condição de contorno térmica de fluxo prescrito nulo.

Palavras-chave: trocador de calor solo-ar, simulação numérica, condições de contorno.

The earth-air heat exchanger (EAHE) is a device used to improve the thermal condition of built environments, contributing to the reduction in electrical energy consumption of traditional air conditioning equipments. So the objectives of this study are to evaluate the influence of the thermal boundary condition of null flux prescribed in the thermal potential (TP) of the TCSA, which is imposed on the sides of the computational domain, and to study the effect of the type of thermal boundary condition imposed on the lower surface of the computational domain in the processing time of an EAHE numerical simulation. To do so, a validated and verified computational model, developed in FLUENT software, which is based on the Finite Volume Method (FVM), was employed. The results showed that the PT of the EAHE installations analyzed is affected for a horizontal spacing between the duct and the computational domain wall, less than $2.00 \mathrm{~m}$. Furthermore, it was found that the use of the boundary condition of prescribed temperature imposed to the lower surface at the computational domain contributed to the reduction of the numerical simulation processing time in $18 \%$, if compared with the use of the boundary condition of prescribed null thermal flux.

Keywords: earth-air heat exchanger, numerical simulation, boundary conditions

\section{INTRODUÇÃO}

Uma instalação de trocador de calor solo-ar (TCSA) consiste em dutos dispostos no subsolo por onde o ar escoa forçado pela ação de ventiladores. Esses dutos são instalados em profundidades relativamente pequenas, possibilitando a troca de calor entre o solo e o ar. Como 
a temperatura das camadas superficiais do solo é sempre mais amena que a temperatura do ar externo ambiente, o ar que sai do TCSA pode ser usado para melhorar a condição térmica de ambientes construídos [1].

Parâmetros operacionais de TCSA, bem como o desempenho térmico do mesmo e as dimensões do domínio computacional foram investigados por meio de modelos analíticos, independentemente do solo e das condições climáticas. Dentre outras análises, os resultados mostram que o TCSA depende de três parâmetros fundamentais: comprimento, diâmetro e número de dutos em paralelo. Também foi observado que o desempenho térmico é melhorado com a redução do diâmetro e aumento do número de dutos em paralelo [2].

Em [3] foi realizado um estudo experimental e numérico sobre o desempenho térmico do TCSA. Foram analisados a condutividade térmica do solo, a velocidade do escoamento e o diâmetro do tubo. Os resultados mostraram que o desempenho térmico de um TCSA depende da condutividade térmica do solo e do tempo de funcionamento do mesmo. Foi constatado que dutos de diâmetro menores requerem que a condutividade térmica do solo seja maior. Foi observado, também, que o aumento de velocidade do fluido leva à diminuição do desempenho térmico do sistema de trocador de calor solo-ar.

O funcionamento do TCSA é influenciado por uma série de parâmetros operacionais, incluindo a configuração geométrica, o comprimento dos dutos, número de dutos em paralelos, propriedades termofísicas dos materiais, área de sombreamento do solo, cobertura da superfície do solo, como também os fatores ambientais. Estudos apontam que tais parâmetros e características afetam significativamente o desempenho térmico do TCSA $[4,5]$.

Para avaliar a eficiência do TCSA, o potencial térmico $(P T)$ é uma variável que vem sendo utilizada como indicador e uma importante referência no estudo do desempenho térmico de TCSA $[6,7,1]$. O PT do TCSA é, basicamente, a diferença da temperatura média na seção de saída de ar no duto e a temperatura média na seção de entrada do ar no duto em relação ao tempo [1].

Dessa forma, os objetivos deste trabalho são de avaliar a influência no $P T$ do TCSA da condição de contorno térmica de fluxo prescrito nulo que é imposta nas superfícies laterais do domínio computacional, e de estudar o efeito do tipo de condição de contorno térmica imposta na superfície inferior do domínio computacional no tempo de processamento da simulação numérica de um TCSA.

A referida proposta se sustenta à medida em que não foram encontrados na literatura trabalhos que contemplem este tipo de investigação numérica. Por isso, o presente trabalho se torna relevante pela tentativa de fornecer informações para a construção de domínios computacionais de TCSA de forma mais criteriosa, a fim de evitar que os mesmos não afetem significativamente os resultados obtidos na simulação numérica de TCSA. Além disso, tentativas eficientes de minimização do tempo de processamento da simulação numérica, em engenharia, são necessárias e importantes para o desenvolvimento e evolução de estudos no campo da Dinâmica de Fluidos Computacional (CFD - Computational Fluid Dynamics).

\section{MATERIAL E MÉTODOS}

Nesta seção foram apresentadas a descrição do problema, as propriedades termofísicas do solo e do ar, o modelo computacional utilizado, bem como a modelagem matemática e numérica para aplicações em TCSA.

\subsection{Descrição do problema}

Neste estudo, por definição, será denominada de Instalação 1 a instalação de TCSA constituída de um único duto retilíneo, com diâmetro $d_{l}=110 \mathrm{~mm}$ e de Instalação 2 a instalação de TCSA também constituída de um único duto, porém com diâmetro $d_{2}=220 \mathrm{~mm}$, sendo que em ambas as instalações o duto é enterrado no solo a uma profundidade de 3,00 m, conforme ilustra a Figura 1. 


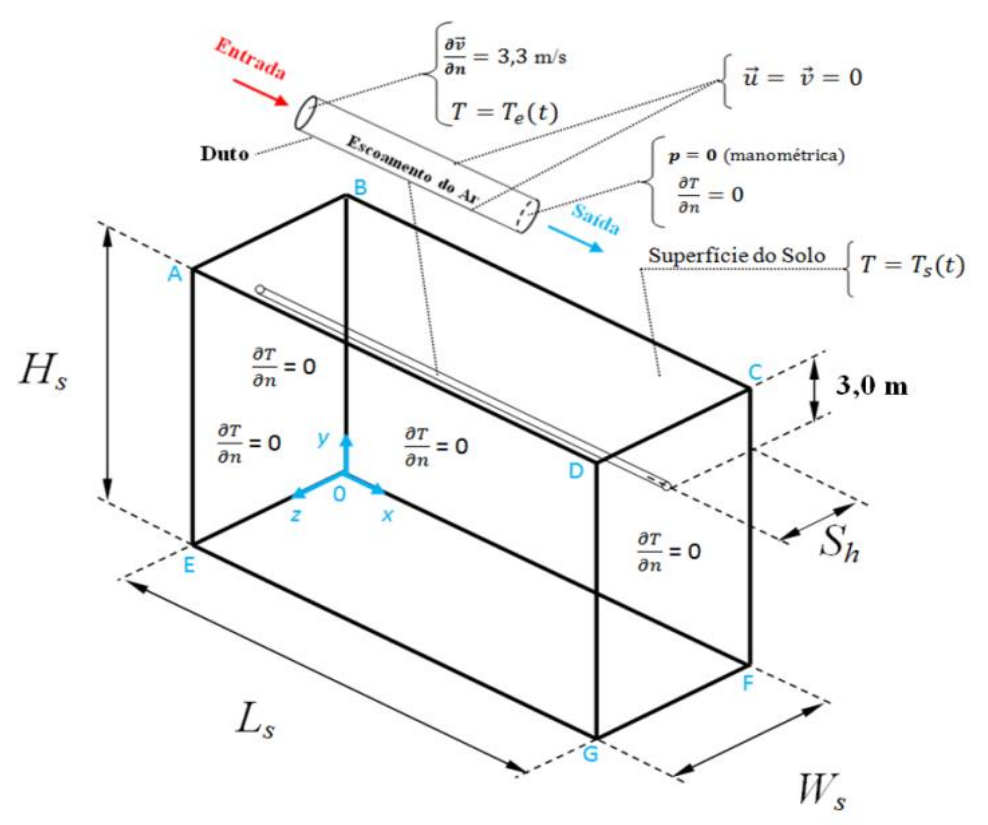

Figura 1: Domínio computacional e condições de contorno do TCSA.

Dessa forma, tem-se o volume de solo representada pelo poliedro indicado por $\overline{\mathrm{ABCDEOFG}}$, onde o comprimento, a largura e a profundidade da porção de solo são, respectivamente, representados por $L_{S}, W_{s}$ e $H_{s}$ assumindo, neste trabalho, os valores iguais a $26,00 \mathrm{~m}, 10,00 \mathrm{~m}$ e $15,00 \mathrm{~m}$. A variável denominada de afastamento horizontal, entre o centro do duto e a superfície lateral do domínio computacional é expressa por $S_{h}(\mathrm{~m})$. As superfícies lateral e inferior do domínio computacional são isoladas termicamente $\left(\frac{\partial T}{\partial n}=0\right)$, onde: $T$ é a variável temperatura (K) e $n$ é a direção normal à superfície analisada. Essa condição de contorno é conhecida como condição de Neumann ou de segunda espécie [8].

As condições de contorno do ponto de vista térmico para a temperatura na superfície do solo e para entrada do ar no duto foram, respectivamente, representadas pelas funções periódicas [7, 9].

$$
\begin{aligned}
& T_{e}(t)=296,18+6,92 \cdot \operatorname{sen}\left(200 \cdot 10^{-9} \cdot t+26,42\right) \\
& T_{s}(t)=291,70+6,28 \cdot \operatorname{sen}\left(200 \cdot 10^{-9} \cdot t+26,42\right)
\end{aligned}
$$

onde: $T_{s}(t)$ é a variável dependente denominada temperatura na superfície do solo $(\mathrm{K}), T_{e}(t)$ é a variável dependente denominada temperatura do ar na seção de entrada do duto (K), $t$ é variável independente chamada tempo (dias), avaliados em um determinado ponto do domínio da função. As Equações (1) e (2) representam uma condição de contorno de temperatura prescrita, conhecida na literatura como condição de Dirichlet, ou de primeira espécie, aplicada no regime transiente [8].

As condição de contorno fluidodinâmicas para entrada e saída do ar no TCSA são respectivamente de velocidade prescrita e pressão prescrita. Assim, para todos os casos analisados a velocidade do ar, na entrada do duto, é igual a 3,30 m/s. Na saída do ar do duto, a condição de pressão prescrita nula foi utilizada, ou seja, o valor da pressão atmosférica normal, do ponto de vista efetiva ou manométrica [1].

Para o escoamento do ar no interior do duto foi considerada as condições de contorno de impermeabilidade e não deslizamento. Do ponto de vista computacional, não foi considerada a espessura da parede do duto de PVC na simulação numérica $[9,10]$.

A Tabela 1 apresenta as propriedades termofísicas adotadas para o solo e para o ar [7, 9]. 
Tabela 1: Propriedades termofísicas do solo e do ar.

\begin{tabular}{cccc}
\hline Componentes & $\begin{array}{c}\text { Massa específica } \\
\rho\left(\mathbf{k g} / \mathbf{m}^{3}\right)\end{array}$ & $\begin{array}{c}\text { Condutividade térmica } \\
k(\mathbf{W} / \mathbf{m} \cdot \mathbf{K})\end{array}$ & $\begin{array}{c}\text { Calor específico } \\
\mathbf{a} \text { pressão constante } \\
c_{p}(\mathbf{J} / \mathbf{k g} \cdot \mathbf{K})\end{array}$ \\
\hline Solo & 1800 & 2,1 & 1.780 \\
$\mathbf{A r}$ & 1,16 & 0,0242 & 1.010 \\
\hline
\end{tabular}

Para avaliar o desempenho térmico do TCSA foi utilizado o potencial térmico médio no tempo e no espaço, chamado de $P T\left({ }^{\circ} \mathrm{C} /\right.$ dia) $)$ O mesmo foi determinado, mensalmente, para cada caso simulado, referente as instalações de TCSA estudadas neste trabalho e é expresso por [1]:

$$
P T=\sum_{i=1}^{t}\left(\frac{\bar{T}_{s_{i}}-\bar{T}_{e_{i}}}{t}\right) \quad(i=1,2,3, \ldots, t)
$$

onde: $\bar{T}_{s_{i}}$ é a temperatura média (K) na seção transversal de saída do ar nos dutos das instalações estudadas, no decorrer do tempo, prevista pela simulação numérica; $\bar{T}_{e_{i}}$ é a temperatura média $(\mathrm{K})$, na seção transversal de entrada do ar nos dutos, no decorrer do tempo, que é dada pela Equação (2) e $t$ é o tempo (dias) relacionando-se com a quantidade de dias do mês analisado. Outras informações sobre o $P T$ podem ser encontradas em [1].

\subsection{Modelagem matemática e numérica}

Primeiramente, é apresentada a modelagem matemática para um TCSA, que consiste nos princípios de conservação da massa, da quantidade de movimento e da energia. Como os escoamentos em TCSA são turbulentos, é necessário também realizar a modelagem da turbulência [1].

A equação de conservação da massa ou equação da continuidade, pode ser escrita na sua forma simplificada da forma $[8,11]$ :

$$
\nabla \cdot \vec{v}=0
$$

onde: $\vec{v}$ é vetor velocidade do escoamento (m/s). Dessa forma, a Equação (4) assume a condição simplificativa de escoamento incompressível e sem fontes de massa [8].

Além disso, para analisar o escoamento do ar no interior do duto, é necessário considerar o princípio fundamental da conservação da quantidade de movimento. Assim sendo, para um sistema de coordenadas retangulares a equação da conservação da quantidade de movimento pode ser apresentada, considerando a modelagem da turbulência, na forma vetorial, por $[8,11$, 12]:

$$
\frac{\partial \rho \overline{\vec{v}}}{\partial t}+\nabla \cdot(\rho \overline{\vec{v} \vec{v}})=-\nabla \bar{p}+\nabla \cdot \overline{(\overline{\bar{\tau}})}
$$

onde: $\rho$ é a massa específica do $\operatorname{ar}\left(\mathrm{kg} / \mathrm{m}^{3}\right) ; p$ é a pressão estática (piezométrica) do fluido $\left(\mathrm{N} / \mathrm{m}^{2}\right)$; e $\overline{\bar{\tau}}$ é o tensor de tensões viscosas relacionada às forças de superfície em um elemento infinitesimal de fluido $\left(\mathrm{N} / \mathrm{m}^{2}\right)$. Dessa forma, a Equação (5) assume a condição de convecção forçada, escoamento turbulento, não havendo forças de campo.

De forma conjunta, as Equações (4) e (5) são utilizadas para determinar o campo de pressão e velocidades do escoamento. Sendo que, na solução, a primeira, atua de forma restritiva à solução do problema.

No que se refere ao tensor de tensões viscosas, o mesmo pode ser expresso por [8,11, 12]: 


$$
\overline{\bar{\tau}}=\mu\left[\left(\nabla \cdot \vec{v}+\nabla \cdot \vec{v}^{T}\right)\right]
$$

onde: $\mu$ é a viscosidade dinâmica ou absoluta do fluido $(\mathrm{kg} / \mathrm{m} \cdot \mathrm{s})$.

Como os fenômenos atinentes ao TCSA envolvem transferência de energia térmica, o princípio fundamental da conservação da energia deve ser respeitado.

Diante disso, é apresentada a equação da energia utilizada para o estudo térmico do escoamento do ar no interior dos dutos. Portanto, a equação da conservação da energia, na forma mais geral, na forma vetorial, considerando a modelagem da turbulência, pode ser expressa por $[8,11,12]$ :

$$
\frac{\partial \rho \bar{h}}{\partial t}+\nabla[\vec{v}(\rho \bar{h})]=\nabla \cdot\left[k_{e f f} \nabla \bar{T}\right]
$$

A entalpia específica, representada pela variável $h$, pode ser expressa por:

$$
h=\int_{T_{r e f}}^{T} c_{p} d T
$$

onde: $c_{p}$ é o calor específico a pressão constante $(\mathrm{J} / \mathrm{kg} \cdot \mathrm{K})$, e $T_{r e f}$ é uma temperatura em um estado termodinâmico de referência $\left(T_{r e f}=298,15 \mathrm{~K}\right)$. Por fim, $k_{e f f}$ é a condutividade efetiva $(\mathrm{W} / \mathrm{m} \cdot \mathrm{K})$ que é dada por $k+k_{t}$, onde $k_{t}$ é a condutividade térmica turbulenta, definida em concordância com o modelo de turbulência adotado.

Para a região do solo, foi resolvida a equação da difusão do calor, que pode ser expressa por

$$
\nabla^{2} T=\frac{1}{\alpha} \frac{\partial T}{\partial t}
$$

onde: $\alpha$ é a difusividade térmica no solo, sendo igual a $65,54 \cdot 10^{-8} \mathrm{~m}^{2} / \mathrm{s}$.

A turbulência foi modelada através do modelo $k-\varepsilon$. O modelo $k-\varepsilon$, resumidamente, envolve a modelagem de duas equações diferencias de transporte:uma para a energia cinética turbulenta $(k)$ e outra para dissipação viscosa turbulenta $(\varepsilon)$. Segundo [12], o modelo $k$ - $\varepsilon$ representa o modelo de duas equações amplamente difundidas, sendo considerado o modelo de turbulência padrão em simulações industriais. Este modelo tem sido extensivamente validado para diversas aplicações, apresentando boa precisão e robustez. Entretanto, o modelo $k-\varepsilon$ não apresenta resultados apropriados para a modelagem de escoamentos livres. Uma das formas encontradas para melhorar os resultados nesses casos é de ajustar as constantes do modelo.

Em [13] o modelo $k-\varepsilon$ foi utilizado satisfatoriamente na modelagem da turbulência em TCSA. Nesse estudo, o presente modelo se mostrou apropriado para aplicações tanto em geometrias retilíneas, quanto em geometrias complexas de instalações de TCSA. Além disso, a utilização do modelo $k-\varepsilon$ garante um menor tempo de simulação em comparação com o modelo RSM utilizado em $[1,7,9]$.

A modelagem numérica foi realizada através de um modelo computacional simplificado [7, 14]. Esse modelo computacional foi validado e verificado com base em [9]. É importante destacar que a simulação numérica, basicamente, é dividida em três etapas: pré-processamento, processamento e pós-processamento. O pré-processamento foi realizado através do software GAMBIT, onde foi construída a geometria do problema, expressa o tipo de condição de contorno e a malha computacional. O software FLUENT realizou o processamento e o pósprocessamento. Esse software é baseado no Método dos Volumes Finitos. No pósprocessamento também foi utilizado o software EXCEL.

A tarefa do método numérico é basicamente transformar uma equação diferencial definida no domínio $D$, em um sistema de equações algébricas. Para tanto, as derivadas das funções existentes na equação diferencial devem ser substituídas pelos valores discretos na função. Assim, transformar as derivadas em termos que contêm a função significa integrar a equação diferencial. Já as diversas formas de fazê-lo, caracterizam o tipo de método numérico [8]. 
O MVF foi utilizado neste trabalho, pois atende, em nível de volumes elementares, os princípios de conservação da massa, do movimento e da energia. Esse método é ferramenta importante no campo da transferência do calor e mecânica dos fluidos computacional (CFD) [8, $11,12]$.

Há, fundamentalmente, duas maneiras de se obter as equações aproximadas no MVF. A primeira é a realização do balanço das propriedades em questão nos volumes elementares, ou volumes finitos. A segunda é integrar sobre o volume elementar, no espaço e no tempo, as equações na forma conservativa. A forma conservativa é aquela em que na equação diferencial os fluxos estão dentro do sinal da derivada e, na primeira integração, aparecem os fluxos nas fronteiras do volume elementar, equivalente, portanto, ao balanço [8].

Neste trabalho, foi utilizado o esquema de advecção upwind para os termos advectivos. Para o presente esquema, a direção do escoamento é levada em consideração para a realização do cálculo do valor de uma determinada variável (velocidade, temperatura) na face do volume finito. Além disso, a positividade dos coeficientes dos termos advectivos é sempre garantida, sendo geradas soluções numéricas ausentes de oscilações numéricas. Aliado a isso, como grande vantagem, se tem que a função de interpolação upwind é a simplicidade de sua aplicação em algoritmos de CFD [7, 9, 14].

Também é importante destacar que, neste trabalho, é utilizado o esquema de avanço temporal totalmente implícito. Nessa formulação, de um modo geral, as incógnitas estarão no mesmo nível de tempo. Assim, tem-se um sistema de equações algébricas para ser resolvido. Para solução transiente é importante resolver o problema com precisão a cada passo de tempo. Dessa forma, se um método iterativo está sendo utilizado, para a resolução do problema, é necessário que os valores das incógnitas sejam iterados até que um critério de convergência seja satisfeito naquele passo de tempo [8].

Neste estudo, foi utilizado o esquema de solução Coupled. O modelo acoplado oferece algumas vantagens com relação ao esquema não acoplado ou segregado, como por exemplo uma implementação robusta. Para escoamentos transientes, é indicado quando a qualidade da malha é baixa ou quando são empregados grandes passos de tempo. O FLUENT disponibiliza quatro algoritmos de acoplamento pressão-velocidade: SIMPLE, SIMPLEC, PISO e Coupled. De um modo em geral, estes utilizam o modelo de solução segregado para o sistema de equações, que devem ser resolvidos, exceto o Coupled, que utiliza o modelo de solução acoplado [1].

A temperatura de inicialização da simulação, para todo o domínio computacional, coincide com a temperatura média do solo, ou seja, $18,7{ }^{\circ} \mathrm{C}(291,7 \mathrm{~K})$. Além disso, nas simulações numéricas, as soluções foram consideradas convergidas quando os resíduos para a equação da massa e para o movimento foram inferiores a $10^{-3}$ e para a energia $10^{-6}$, entre duas iterações consecutivas. Além disso, foi realizado o número máximo de 200 iterações por passo de tempo para convergência dos resultados. Também é importante frisar que para todas as simulações numéricas utilizou-se um passo de tempo de $3.600 \mathrm{~s}$ e 17.520 passos de tempo. Essa configuração permite simular dois anos de comportamento térmico e fluidodinâmico em instalações de TCSA. No entanto, foram analisados apenas os dados referentes ao segundo ano, sendo desconsiderados os dados referentes ao primeiro ano $[1,6,7,9,14]$.

\section{RESULTADOS E DISCUSSÃO}

A primeira proposta consistiu na obtenção dos dados numéricos, que foram gerados por meio de dois casos distintos: o Caso 1 foi constituído de 11 simulações numéricas referentes à Instalação 1, enquanto o Caso 2, referiu-se à Instalação 2 e constituiu-se do mesmo número de simulações realizadas para Instalação 1 . Em ambos os casos o valor assumido pela variável $S_{h}$ esteve sujeito às seguintes restrições geométricas: $S_{h} \leq 5,00 \mathrm{~m}$ e $S_{h} \geq d_{i}$, onde: $d_{i}$ é o diâmetro do duto $(\mathrm{mm})$, sendo $i=\{1,2\}$. Nesse estudo, o objetivo foi de avaliar a influência da condição de contorno térmica de $\left(\frac{\partial T}{\partial n}=0\right)$ imposta nas superfícies laterais do domínio computacional no $P T$ do TCSA. 
Para exposição dos resultados, apenas os meses de Janeiro, Junho, Julho, Agosto, Novembro e Dezembro foram considerados, já que nos demais meses do ano, os valores de $P T$ não se mostraram com variações significativas. Além disso, também se mostraram intermediários em relação aos meses de Dezembro e Junho, os quais apontaram os maiores valores de $P T$ de resfriamento e aquecimento, respectivamente.

A Figura 3 mostra a variação do $P T$ em função de $S_{h}$ para a Instalação 1 (Caso 1). É possível notar que para $S_{h}<2,00 \mathrm{~m}$, o $P T$ é influenciado pela condição de contorno lateral do domínio computacional de fluxo prescrito nulo. No entanto, para $S_{h} \geq 2,00 \mathrm{~m}$, o $P T$ não é afetado significativamente pela condição de contorno especificada. Além disso, a maior variação do $P T$ se deu para o mês de Dezembro, sendo igual a $-0,83^{\circ} \mathrm{C} / \mathrm{dia}$, o que corresponde a $9,76 \%$.

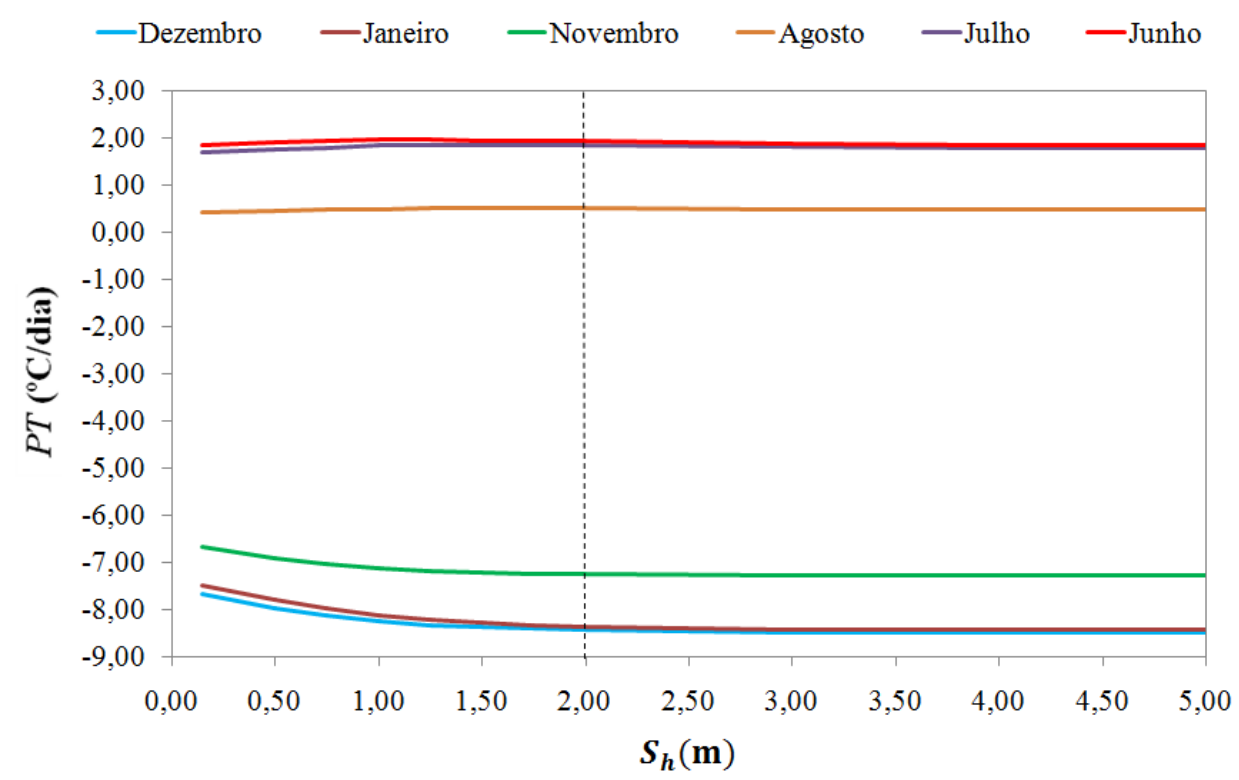

Figura 3: Variação do PT em função de $S_{h}$ para a Instalação 1.

A Figura 4 apresenta, para a Instalação 2, a variação do $P T$ em função de $S_{h}$ (Caso 2). É possível notar que para $S_{h} \geq 2,00 \mathrm{~m}$, o $P T$ não é afetado de modo significativo pela condição de contorno de fluxo prescrito nulo. Entretanto, para $S_{h}<2,00 \mathrm{~m}$, o $P T$ é influenciado pela condição adiabática. Analisando os resultados foi possível observar que a maior variação do $P T$ se deu também para o mês de Dezembro, sendo igual a $-0,78^{\circ} \mathrm{C} /$ dia, o que corresponde a $18 \%$ do $P T$ para este período.

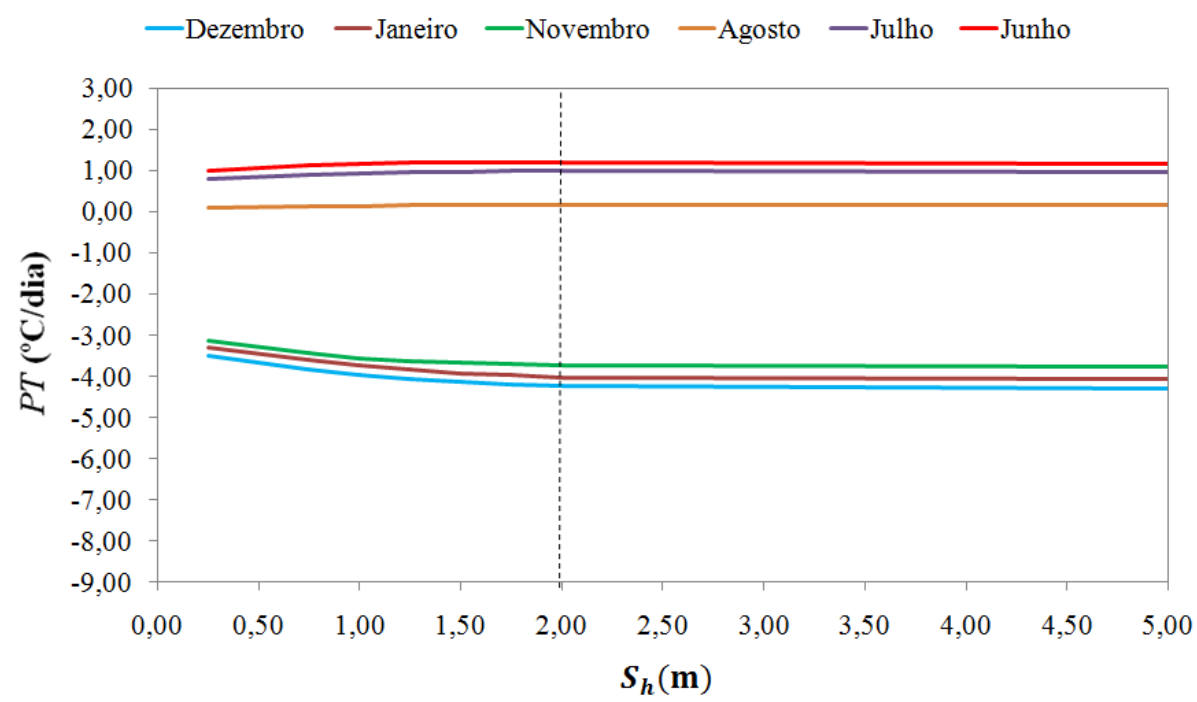

Figura 4: Variação do PT em função de $S_{h}$ para a Instalação 2. 
Também foi possível observar, através das Figuras 3 e 4, que a variação do diâmetro não acarretou em significativa oscilação no comportamento das curvas de PT do TCSA para os casos analisados. Também foi observado que, para ambos os casos simulados, o PT do TCSA foi influenciado para $S_{h}<2,00 \mathrm{~m}$.

A Figura 4 mostra a variação do $P T$ em função de $S_{h}$ para a Instalação 2. É possível verificar uma tendência muito semelhante em comparação com a ocorrida para a Instalação 1. Dessa forma, para valores de $S_{h} \geq 2,00 \mathrm{~m}$, o $P T$ permanece praticamente constante em relação ao $S_{h}$. No entanto, para valores de $S_{h}<2,00 \mathrm{~m}$ tal tendência não se confirma. Obviamente, outros casos de instalações de TCSA contendo diferentes diâmetros devem ser investigados a fim de estabelecer um critério geral para determinação de $\left(S_{h}\right)_{\min }$ (espaçamento mínimo na horizontal), entre o duto e a parede do domínio computacional, de tal forma que não haja influência da condição de contorno adiabática no $P T$ na modelagem de TCSA. Dessa forma, para ambos os casos analisados, foi obtido $\left(S_{h}\right)_{\min }=2,00 \mathrm{~m}$.

A segunda proposta deste trabalho visou investigar a influência do tipo de condição de

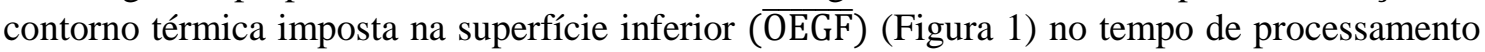
para a simulação numérica de TCSA. O modelo computacional utilizado estabelece a condição de contorno térmica de $\left(\frac{\partial T}{\partial n}=0\right)$ para a superfície inferior mencionada anteriormente. Os estudos $[9,15]$ mostram que para $H_{S} \geq 15,00 \mathrm{~m}$ a temperatura do solo é praticamente constante em $18,7^{\circ} \mathrm{C}$.

A temperatura prescrita, é a condição de contorno na qual a temperatura da face analisada assume um valor pré-determinado, podendo o mesmo ser constante ou variável no tempo. Fisicamente, por exemplo, o caso de temperatura prescrita constante pode ser obtida através do contato físico de dado material com um segundo material na temperatura de mudança de fase. Assim, a temperatura de interface entre os dois materiais pode ser considerada constante. Neste estudo, sabendo-se que, para que para $\mathrm{H}_{\mathrm{s}} \geq 15,00 \mathrm{~m}$, a temperatura é constante (nas camadas superficiais da crosta terrestre), logo é possível inferir que a temperatura no ponto sobre uma superfície ortogonal à direção da profundidade é prescrita e constante. Já o fluxo prescrito consiste na condição de contorno na qual o calor trocado entre os meios materiais estudados assume um valor pré-determinado. Essa quantidade pode ser constante ou variável em função do tempo. O caso de fluxo prescrito, por exemplo, pode ser obtido experimentalmente através da radiação de uma fonte conhecida e controlável em ambiente de vácuo. Neste estudo, a utilização da condição de contorno de fluxo prescrito nulo garante que não há taxa de transferência de energia térmica no tempo, isto é, dá-se a imposição de uma superfície perfeitamente isolada termicamente (face adiabática) [1,8].

Portanto, para essa análise, também foram definidos dois casos de estudo: o Caso 3 consistiuse de uma simulação numérica de TCSA realizada para as condições definidas para a Instalação 1 utilizando a condição de contorno adiabática para a superfície inferior do domínio computacional. Já o Caso 4 foi constituído de uma simulação numérica realizada com as definições referentes à Instalação 1 , exceto para a aplicação da condição de contorno de temperatura prescrita $\left(T_{s}=18,7^{\circ} \mathrm{C}\right)$ paras a superfície $(\overline{\mathrm{OEGF}})$. Para ambos os casos foram simulados dois anos, no entanto, para análise dos resultados foi considerado apenas os dados numéricos referentes ao segundo ano [1]. Dessa forma, para garantir a qualidade dos resultados foi utilizado como indicador para comparação do desempenho térmico do sistema o $P T$ do TCSA.

A Figura 5 mostra o comportamento do $P T$, para resfriamento e aquecimento, em função do tempo, para as condições de contorno analisadas. É possível notar que não há signifícativa variação entre o comportamento do $P T$ entre os Casos 3 e 4 analisados. Dessa forma, a máxima variação do $P T$ se deu para o resfriamento sendo igual a $0,43^{\circ} \mathrm{C} /$ dia para o período de Janeiro. Com isso, foi observado que a utilização da condição de contorno de temperatura prescrita (Caso 4) não influenciou de modo significativo nos resultados numéricos. 


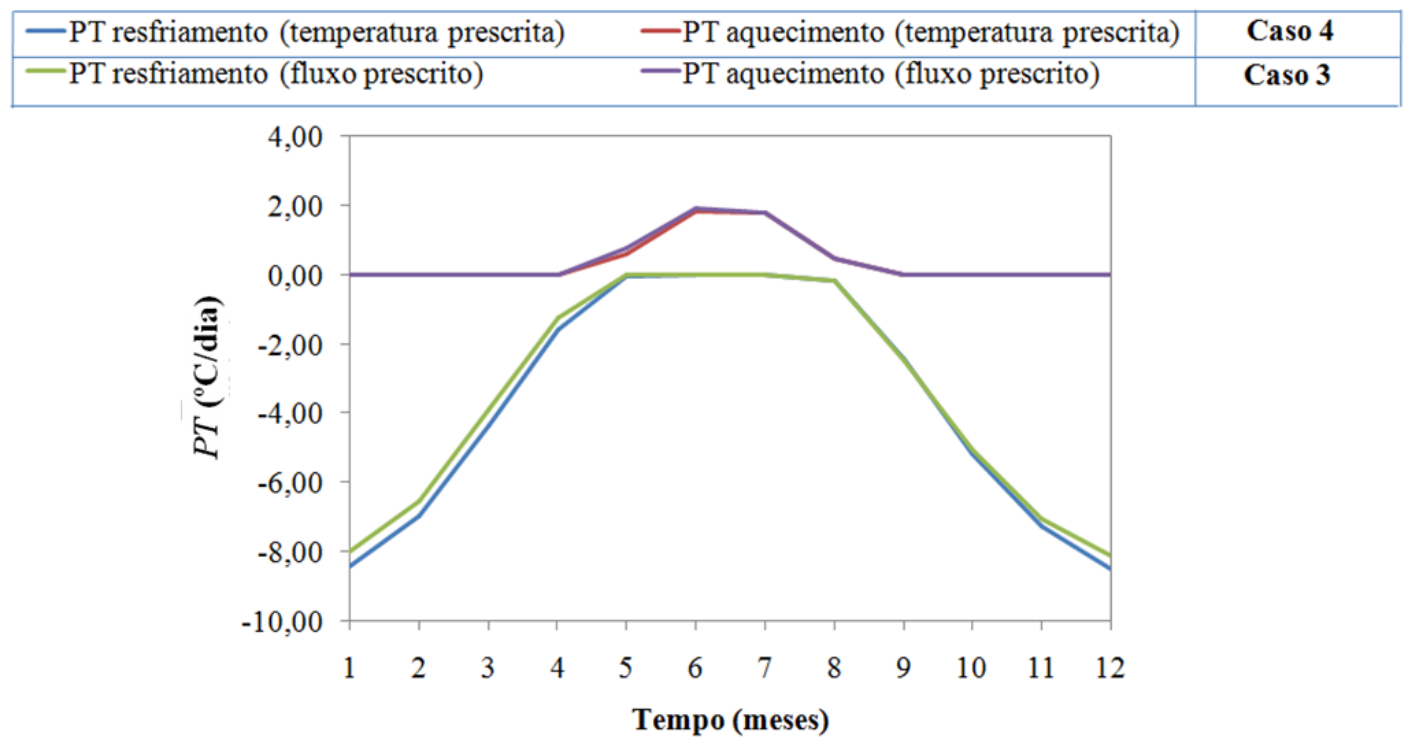

Figura 5: Comportamento do PT em função do tempo para a Instalação 1.

Entretanto, por meio da modelagem especificada no Caso 4, quando comparada com o Caso 3 , foi possível reduzir satisfatoriamente o tempo de processamento da simulação numérica em aproximadamente $18 \%$ conforme pode ser visualizado na Tabela 2.

Tabela 2: Tempo computacional em função da condição de contorno térmica para a superfície inferior do domínio computacional referente à Instalação 1 .

\begin{tabular}{ccc}
\hline & \multicolumn{2}{c}{ Condição de contorno x tempo computacional } \\
\cline { 2 - 3 } Caso analisado & Condição de contorno & Tempo computacional \\
\hline Caso 3 & Fluxo prescrito $\left(\frac{\partial \mathrm{T}}{\partial \mathrm{n}}=0\right)$ & $10 \mathrm{~h} \mathrm{e} 33 \mathrm{~min}$. \\
Caso 4 & Temperatura prescrita $\left(T_{S}=18,70^{\circ} \mathrm{C}\right)$ & $8 \mathrm{~h} \mathrm{e} 41 \mathrm{~min}$. \\
\hline
\end{tabular}

Obviamente, a imposição da condição de contorno de $T_{s}=18,7^{\circ} \mathrm{C}$ (Caso 4), isto é, um valor constante é viável apenas para domínios computacionais com $H_{s} \geq 15,00 \mathrm{~m}$.

\section{CONCLUSÃO}

Em suma, as finalidades deste trabalho foram de analisar a influência da condição de contorno térmica de fluxo prescrito nulo, imposta nas superfícies laterais do domínio computacional, no $P T$ do TCSA e de avaliar o efeito do tipo de condição de contorno térmica imposta na superfície inferior do domínio computacional no tempo de processamento da simulação numérica de um TCSA.

Os resultados indicam que o $P T$ é influenciado para o afastamento horizontal $\left(S_{h}\right)$ menor do que 2,00 $\mathrm{m}$ para as instalações de TCSA analisadas. No entanto, essa variação no $P T$ foi menor do que $1,00^{\circ} \mathrm{C} /$ dia para os casos simulados. Esse fato se deu por conta do efeito da condição de contorno térmica de fluxo prescrito nulo que foi imposta às superfícies laterais do domínio computacional. Essa indicação servirá de critério construtivo de domínios computacionais na modelagem de TCSA.

Além disso, foi observado através dos resultados que a condição de contorno térmica de temperatura prescrita imposta para a superfície inferior do domínio computacional acarretou em um menor tempo de processamento na simulação numérica para a instalação de TCSA analisada, quando comparada com a aplicação da condição de contorno adiabática aplicada na mesma superfície. Esse ganho de tempo foi de aproximadamente $18 \%$. 


\section{AGRADECIMENTOS}

Os autores agradecem ao CNPq pelo apoio.

\section{REFERÊNCIA BIBLIOGRÁFICAS}

1. Rodrigues M. Modelagem computacional aplicada à melhoria do desempenho térmico de trocador de calor solo-ar Através do método Constructal Design. Dissertação de Mestrado, Universidade Federal do Rio Grande, Programa de Pós-Graduação em Modelagem Computacional (PPGMC). 2014.

2. Paepe MD, Janssens AA. Thermo-hydraulic design of earth-air heat exchangers. Energy and Buildings. 2011;35:389-397.

3. Misra R, Bansal V, Agrawal GD, Mathur J, Aseri TK. CFD analysis based parametric study of derating factor for earth air tunnel heat exchanger. Applied Energy. 2013;103:266-277.

4. Peretti C, Zarrella A, Carli M, Zecchin R. The design and environmental evaluation of earth-to-air heat exchangers (EAHE). A literature review. Renewable and Sustainable Energy Reviews. 2013;28:107-116.

5. Trzaski A, Zawada B. The influence of environmental and geometrical factors on air-ground tube heat exchanger energy efficiency. Building and Environment. 2011;46:1436-1444.

6. Isoldi LA, Brum RS, Rodrigues MK, Ramalho JVA, Vaz J, Sousa JA, dos Santos ED, Rocha LAO. Constructal Design of Earth-Air Heat Exchangers. Constructal Law Conference. Proceedings of Constructal Law Conference, Nanjing. 2013. Vol. 1. pp. 88-96.

7. Brum RS, Rocha LAO, Vaz J, dos Santos ED, Isoldi LA. Development of simplified numerical model for evaluation of the influence of soil-air heat exchanger installation depth over its thermal potential. International Journal of Advanced Renewable Energy Research. 2012;1:505-514.

8. Maliska C. Transferência de Calor e Mecânica dos Fluidos Computacional. Livros Técnicos e Científicos Editora S.A., Rio de Janeiro; 2004. 453 p.

9. Vaz J, Sattler MA, dos Santos ED, Isoldi LA. Experimental and Numerical Analysis of an Earth-Air Heat Exchanger. Energy and Buildings. 2011;43:2476-2482, doi:10.1016/j.enbuild.2011.06.003.

10. Bansal V, Misra R, Agrawal G, Mathur J. Performance analysis of earth-pipe-air heat exchanger for summer cooling. Energy and Buildings. 2010;42:645-648.

11. Patankar S. Numerical Heat Transfer and Fluid Flow. McGraw-hill, New York, USA. 1980.

12. Versteeg H, MAlalasekera W. An Introduction to Computational Fluid Dynamics: The Finite Volume Method. England: Pearson Education Limited, 2a ed.; 2007. 503 p.

13. Kipper R. Análise Numérica do Potencial Térmico de Trocadores de Calor Solo-Ar para Diferentes Configurações de Instalação. Trabalho de Conclusão de Curso de Engenharia Mecânica, Universidade Federal do Rio Grande (FURG), Escola de Engenharia, Rio Grande, RS, Brasil. 2014.

14. Brum R, Vaz J, Rocha LAO, dos Santos E, Isoldi L. A new computational modeling to predict the behavior of earth-air heat exchangers. Energy and Buildings. 2013;64:395-402, doi:10.1016/j.enbuild.2013.05.032.

15. Brum R, Isoldi L, dos Santos E, Vaz J, Rocha LAO. Two-dimensional computational modeling of the soil thermal behavior due to the incidence of solar radiation. $14^{\circ}$ Brazilian Congress of Thermal Sciences and Engineering, Rio de Janeiro; 2012. p.18-22. 